EMIS 2015 NIM-B Proc.

\title{
Recent Results from the TwinSol Low-energy RIB Facility $\quad$ 8/12th/2015
}

\author{
F.D.Becchetti \\ U. Michigan, Ann Arbor MI 48109 \\ J.J. Kolata \\ U. Notre Dame, Notre Dame, IN 46556 \\ and the TwinSol Collaboration
}

KEYWORDS: Radioactive ion beams; unstable nuclear beams; solenoid spectrometer

\begin{abstract}
We report on some of the recent developments and experimental work done at the twin-solenoid low-energy radioactive-ion-beam (RIB) facility TwinSol installed at the U Notre Dame 10 MV FN tandem accelerator. The TwinSol facility is a joint project of the University of Michigan (UM) and the University of Notre Dame (UND), and includes several U.S. and foreign collaborators. A number of significant experiments including RIB-induced transfer reactions, elastic scattering, resonant scattering, and fusion at energies near and well below the Coulomb barrier have been performed with this facility. Several of these as well as future work and upgrades planned will be described.
\end{abstract}

Introduction

The original UM-UND low-energy RIB facility (LilSol) was developed and used from 1987 through 1995 utilizing a $20 \mathrm{~cm}$ bore, $3.5 \mathrm{~T}$ DoE-surplus superconducting solenoid supplied by UM. NSF grants were then obtained to upgrade the UND tandem ion source to produce the high-intensity primary beams needed for RIB production. A nsec beam pulser and pulse-selection system also was added for RIB time-of-flight (ToF) capability. Since fragmentation is not efficient at low energies for RIB production, the RIBs were instead typically produced using selective high cross section, forward-peaked nucleon transfer reactions. This combined with the large acceptance of the solenoid to collect and focus secondary RIBs especially in inverse kinematics, resulted in the production of many useable viz. $10^{4} / \mathrm{s}$ to $>10^{6} / \mathrm{s}$ RIBs near the line of nuclear stability, $A<20$. Despite the poor energy resolution (ca. 0.5-1 MeV), many of the first low-energy RIB-induced reactions, elastic scattering and LE RIB fusion studies were done with this facility. Likewise techniques were developed to efficiently produce short-lived isomeric nuclear beams again using selective nuclear reactions. This technique was later used at the MSU NSCL facility to study elastic scattering of protons from a nucleus $\left({ }^{18} \mathrm{~F}^{\mathrm{m}}\right)$ in a high-spin $\left(5^{+}\right)$excited state as well as measurements of nuclear reaction cross sections for an excited, isomeric nucleus, the first of this type measurement. Other work pioneered measurements of near-barrier fusion and transfer reactions using neutron- and proton-rich RIBs including a number of key experiments related to nuclear astrophysics. Several measurements using implanted RIBs also were done, including use of a high-resolution cryogenic EC x-ray detector. A listing of the published work and related theses describing and using this facility can be found at the TwinSol publication web site (Ref 1) for the years 1987-1995.

The TwinSol facility (1996-2015)

A major upgrade of the UM-RIB facility was proposed and funded by the NSF with UM and UND matching funds in 1993. This involved the design and construction of a pair of $30 \mathrm{~cm}$ bore, $6 \mathrm{~T}$ superconducting solenoids (Figs.1-3) together with upgrades to the UND FN tandem and the UND facilities. To minimize neutron activation issues and facilitate the installation and possible future re-configuration of the magnets (see below), the solenoid cryostats and beam line 
components were primarily made of aluminum. As UND did not have a LHe recovery system, the magnets utilize a low-loss cryostat design $(<0.1 \mathrm{I} \mathrm{LHe/hr})$ with $>2$ month hold time, and usually operate in persistent mode. Likewise there are no steel yokes and hence the magnets easily can be tuned or re-tuned for a specific RIB rather quickly as the field scales exactly with the magnet current. The solenoids are a multi-coil design with extensive quench protection. However should a coil be damaged during a quench it can be shorted i.e. bypassed via the internal coil circuitry and the solenoid remain operational (see IEEE papers and M.Lee thesis in Ref.1).

The magnets can be operated with or without a beam cross over between them (Fig.3) and both modes have been used depending on the particular experiment. The cross over focus mode provides a suitable location that can be utilized for a timing detector or more typically an energy loss absorber. The latter together with the second solenoid helps to remove some of the unwanted analog RIBs, especially on the neutron-rich side. The first RIB experiments started in 1996 using a single magnet and a small scattering chamber located at a focal point between the magnets. The second magnet was designed to be used as part of the RIB production system, or alternately as a spectrometer for RIBs produced by the first magnet (see below)

Initially the two magnets were located in the West Target Hall at UND (Fig. 2) with a small scattering chamber ( $0.5 \mathrm{~m}$ diameter) normally located behind the second magnet (among several possible RIB focal positions along the solenoids' axes). Several important low-energy RIB experiments were done using this arrangement, many with U.S. and foreign collaborators as the lead scientists (see Ref.1).

In order to permit better long-path ToF (>10 m) and low-b.g. measurements involving detection of neutrons, a new experimental area together with an intervening shielding wall using boronloaded water was constructed permitting RIB experiments to be done in a low b.g. room several meters beyond the last solenoid (Figs. 2 and 3 ). This also involved construction of a new, large (1 $\mathrm{m}$ dia.) moveable scattering chamber that could be used with a variety of large-area detectors. Alternately smaller chambers have been used at appropriate RIB focal locations in the low b.g. room, especially for neutron measurements. To facilitate the latter, a large-area plastic-scintillator neutron wall and cosmic-ray veto system was built. Break-up and transfer reactions of the neutron-rich nucleus ${ }^{6} \mathrm{He}$ near the Coulomb barrier were studied with this neutron wall, often in coincidence with an outgoing reaction product (Ref.2). Likewise a search for a proposed excited state in ${ }^{7} \mathrm{He}$ was conducted using the ${ }^{6} \mathrm{He}+\mathrm{p}$ reaction near the barrier with negative results (Ref.3).

A number of transfer reactions also were investigated using the upgraded facility. This included study of the ${ }^{12} \mathrm{C}\left({ }^{7} \mathrm{Be},{ }^{3} \mathrm{He}\right){ }^{16} \mathrm{O}$ reaction (Ref.4). As anticipated the $\left({ }^{7} \mathrm{Be},{ }^{3} \mathrm{He}\right)$ reaction proved to be advantageous as a direct alpha-transfer reaction compared to more conventional stable-beam induced alpha-transfer reactions in populating alpha-cluster states with minimal projectile break up and other background. Again, the measurements were limited by the poor energy resolution but this should be greatly improved with the next-generation RIB facilities as will many of the other measurements initially done at TwinSol. Several other interesting experiments involving 7Be also were completed, many with collaborators as the lead scientists (see Ref.1).

Among the more interesting RIBs developed and exploited is the proton-halo $\mathrm{RIB}^{8} \mathrm{~B}$, produced via the ${ }^{6} \mathrm{Li}\left({ }^{3} \mathrm{He}, \mathrm{n}\right)$ reaction done in reverse kinematics. This permitted many measurements to be done at and below the Coulomb barrier, including study of break-up and fusion reactions. Again references to the appropriate work can be found in Ref. 1.

More recently, several measurements have been done utilizing the prototype active-target timeprojection chamber (AT-TPC; Fig.4; Ref.5) built by the TPC group at Michigan State University (MSU). Using ${ }^{4} \mathrm{He}$ as an active-target TPC gas, resonant alpha-particle scattering, fusion and transfer reactions utilizing incident ${ }^{6} \mathrm{He}$ and ${ }^{10} \mathrm{Be} \mathrm{RIBs}$ were measured over a range of energies. Subsequent break up of unusual types of alpha-cluster states in ${ }^{10} \mathrm{Be}$ and ${ }^{14} \mathrm{C}$ were tracked as a function of energy with interesting results (Fig.4) including evidence for a linear 3-alpha $+2 n$ 
cluster state in ${ }^{14} \mathrm{C}$. These and additional experiments will be reported at this conference (Ten Ahn, et al.)

As part of a recent UM PhD project (Ref.6), a deuterated liquid scintillator array was developed (Fig.6) and tested with stable beams at UND including measurement of several $(d, n)$ reactions in light nuclei. This type of scintillator (Eljen 315) can provide useable neutron energy spectra without need for long-path ToF and the array can be configured to provide large angular coverage (Fig.6). This results in a $\times 100$ or more increase in over- all detection efficiency relative to a conventional long-path ToF set up and should prove valuable in RIB experiments such as $(d, n)$. We have verified this with a preliminary measurement of the ${ }^{7} \mathrm{Be}(\mathrm{d}, \mathrm{n})$ reaction (Fig.7) and additional measurements are planned.

Recently, useable RIBs developed include proton-rich ${ }^{10} \mathrm{C},{ }^{17} \mathrm{~F}$ and neutron-rich ${ }^{17} \mathrm{~N}$. Experiments using these are underway.

Future upgrades

At the moment, owing to the magnification of the secondary RIB with the existing magnet configuration (Fig.3), a somewhat large beam spot (several $\mathrm{cm}$ diameter) is produced at the back scattering chamber. While many experiments can accommodate such a large RIB beam spot, it becomes a problem when using the AT-TPC, when good angular resolution is required, or when enriched -isotope targets are needed. Thus it is planned to rearrange the magnets to achieve a smaller RIB beam spot (1 cm dia. or less) at the back focus. The magnets are designed for this as they are mounted on an optical bench arrangement (Figs.1 and 3) and are relatively low weight being air-core magnets with aluminum cryostats (Fig.1). As part of this project a new RIB production chamber is being built which will include multiple target locations including several gas cells. Due to the intense primary beam currents it is now often necessary to replace gas cell windows during a run and having multiple gas cells in place will eliminate that issue.

As noted the second solenoid in the TwinSol set up was designed to be used as spectrometer if appropriate for RIBs produced by the first magnet and focused at the cross-over location or elsewhere on axis. To this end a group at UND is developing and has tested a Helios-type (Ref.7) axial silicon-detector array for insertion in the second solenoid. Initial tests using a subset of detectors was successful and the full array is now under construction.(see paper by P. O'Malley et al. for details on this and other planned upgrades).

\section{Future ReA Experiments}

While the TwinSol facility can be considered to be a 2nd generation RIB facility, experiments are still limited by the low intensity (though high relative to many other LE RIB facilities), limited mass range $(A<20)$, and the relatively poor energy resolution and optical properties of the secondary RIBs. These properties should be greatly improved with the new 3rd generation RIB facilities coming into operation such as the ReA facility at MSU, the upgraded LE RIB facility at TRIUMF, etc. In fact, many of the experiments and tests being done at TwinSol, e.g. the recent work with the prototype AT-TPC, are intended to be predecessors for future, improved experiments to be done at ReA and elsewhere. Yet, TwinSol can still provide certain low-mass RIBs at intensities not likely available at these new facilities and should remain a viable LE RIB facility well into the future, especially as in the past for the hands-on training and education of the next generation of "exotic beam" scientists.

\section{Acknowledgemnents}

We thank the UND technical staff for their assistance and the many collaborators 
who have worked at the LilSol and TwinSol facilities. The research and RIB-related apparatus built by UM and UND have been funded primarily by grants from the U.S. National Science Foundation.

\section{References}

Ref. 1: http://research.physics.Isa.umich.edu/twinsol/Publications/publications.html

Ref. 2: J. J. Kolata, et al., Physical Review C 75 (2007) 031302(R).

Ref.3: G.V. Rogachev, et al., Physical. Review. Letters 92 (2004) 232502.

Ref.4: H. Amro, et al., European Physics Journal Special Topics, 150 (2007) 1.

Ref.5: W. Mittig, et al., Nuclear Instruments and Methods in Physics Research A 784 (2015) 494.

Ref.6: "A Deuterated Neutron Detector Array for the Study of Nuclear Reactions with Stable and Rare Isotope Beams," Michael T. Febbraro ( Ph.D. thesis, The University of Michigan, Applied Physics 2014).

Ref. 7: A. H. Wuosmaa, J. P. Schiffer, B. B. Back, C. J. Lister, and K. E. Rehm, Nuclear Instruments and Methods in Physics Research A 580 (2007)n 1290 


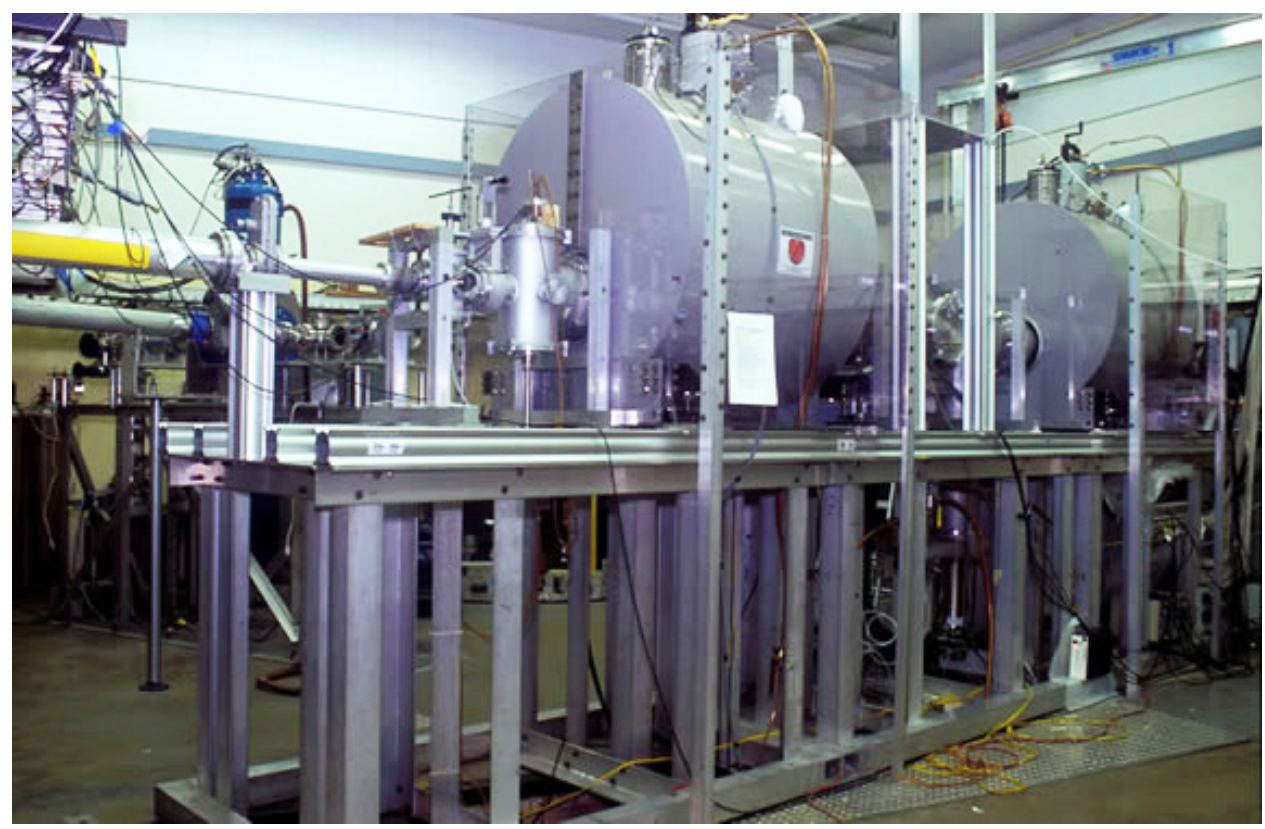

Fig. 1: TwinSol magnet and chamber arrangement installed at UND

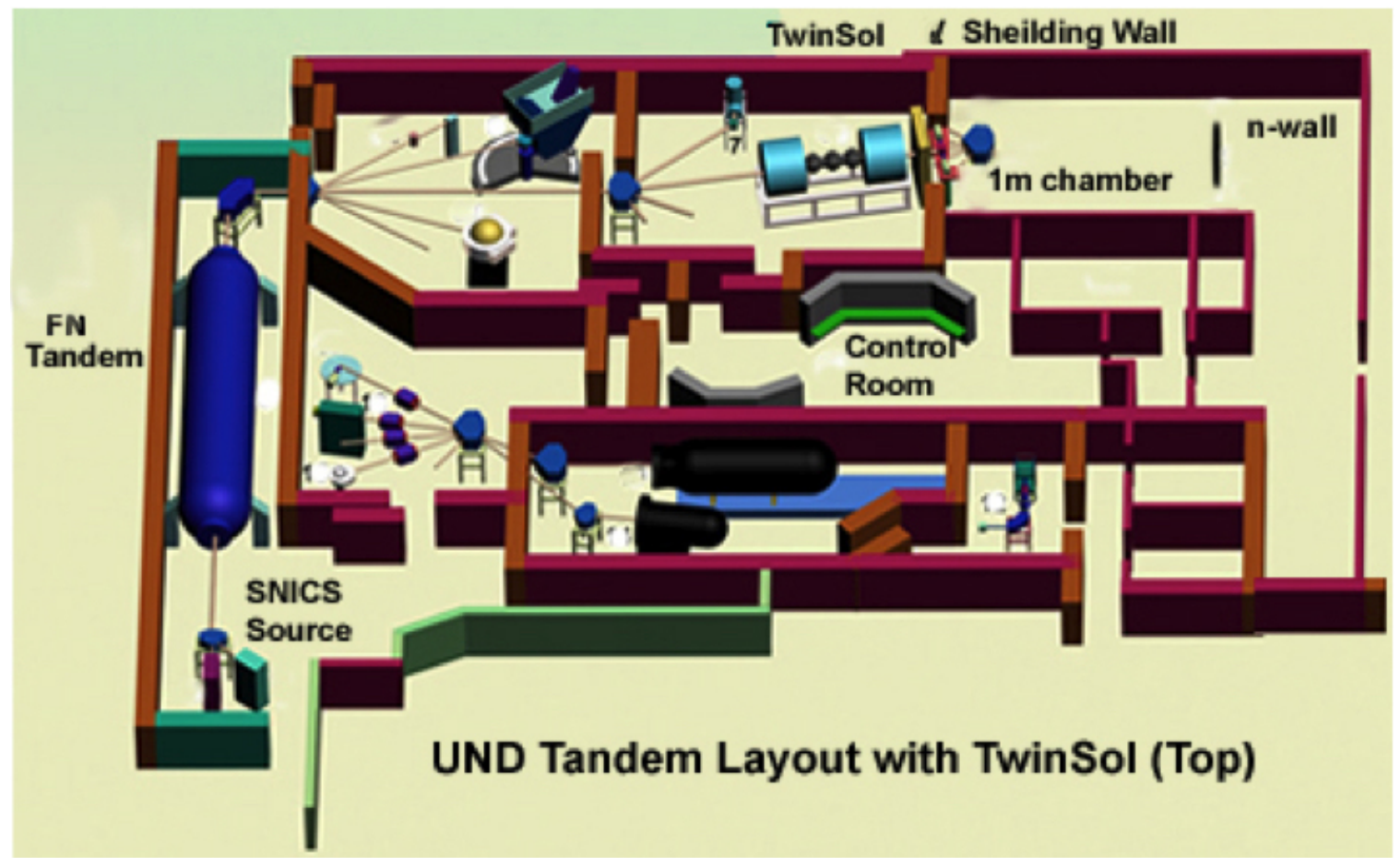

Fig.2: TwinSol layout at the UND FN tandem accelerator. 

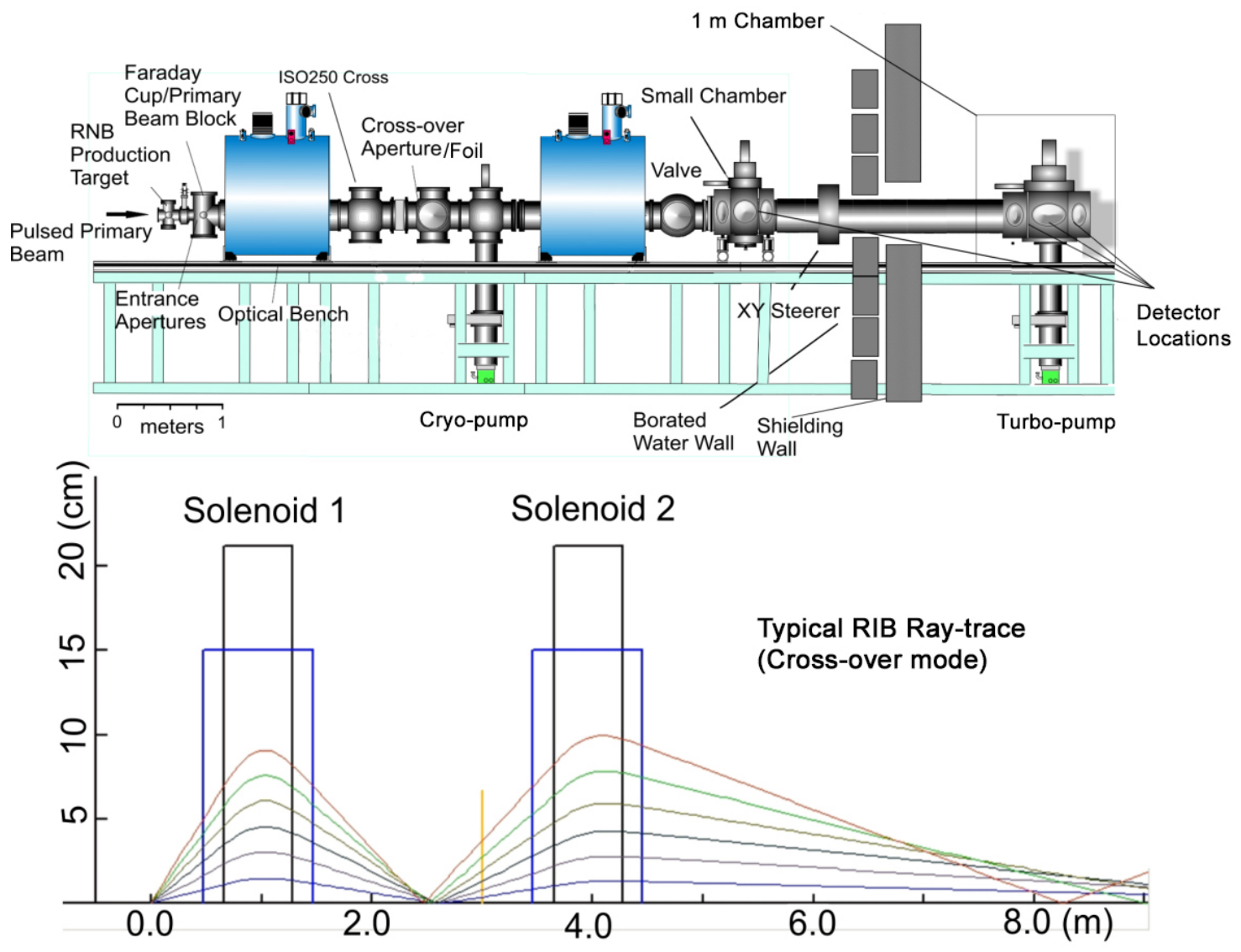

Fig. 3: Present TwinSol configuration and typical RIB ray-trace to $1 \mathrm{~m}$ chamber.

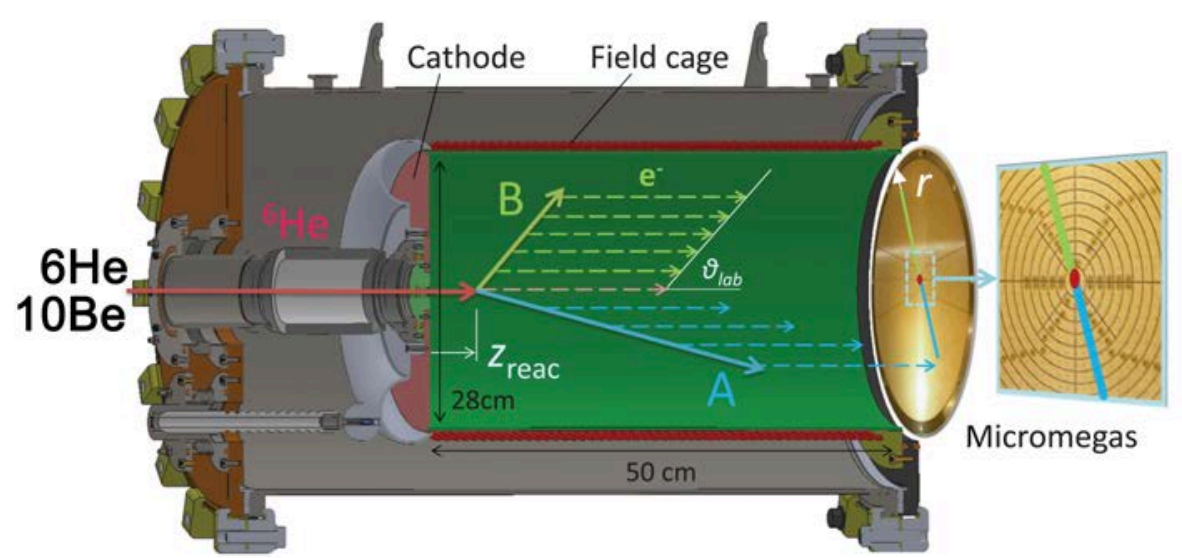

Fig.4: MSU prototype AT-TPC used at TwinSol (Ref.5) 

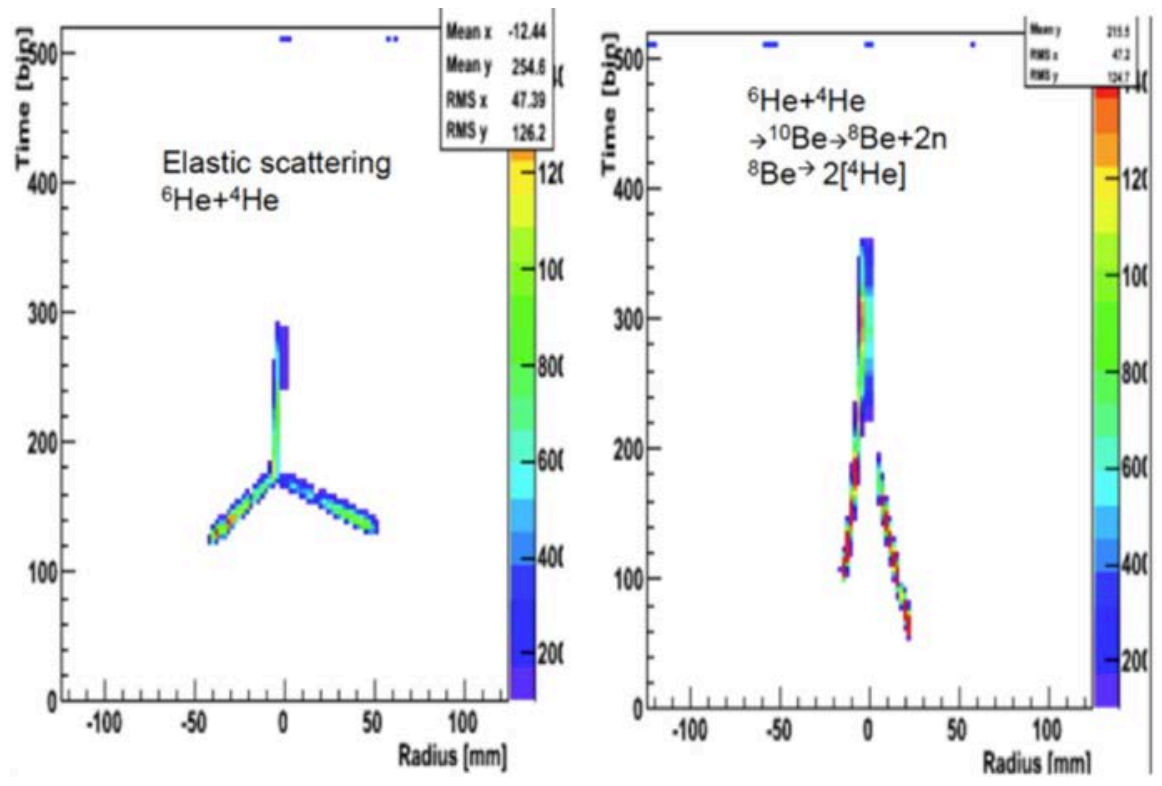

Fig.5: Typical track data observed in the AT-TPC (Fig.3) with incident ${ }^{6} \mathrm{He}$ RIB.

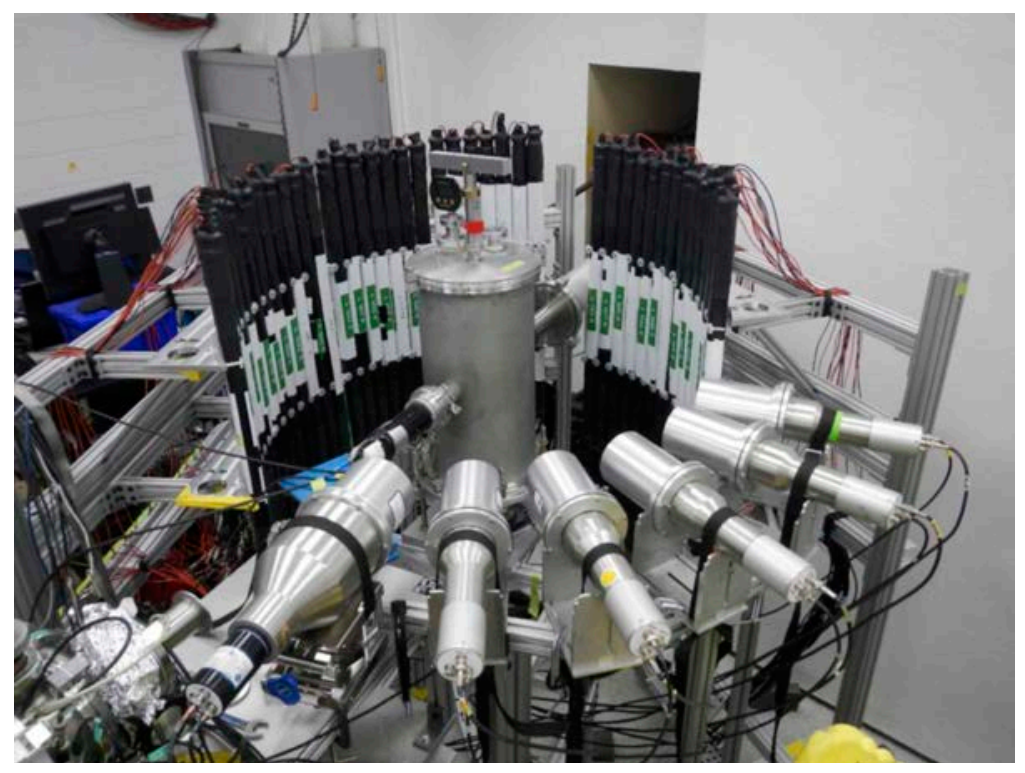

Fig.6: UM deuterated liquid scintillator neutron detector array (bottom) mounted with the ORNL Vandle plastic scintillator neutron-wall at TwinSol (Ref.6). 


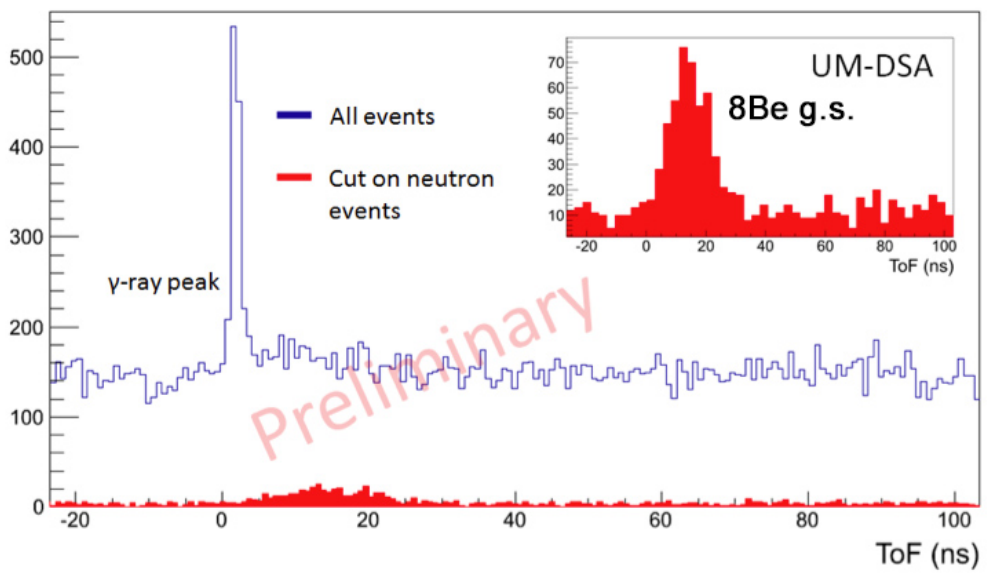

Fig. 7: Preliminary ${ }^{7} \mathrm{Be}(\mathrm{d}, \mathrm{n})^{8} \mathrm{Be}$ g.s. data $(\mathrm{E}=34 \mathrm{MeV})$ obtained with the UM array (Fig. 6; from Ref. 6). 